\title{
Primary ovarian diffuse large B-cell lymphoma preceded by autoimmune hemolytic anemia
}

\author{
Satoshi Ichikawa ${ }^{1}$ (D) - Asami Sasaoka ${ }^{1}$. Noriko Fukuhara ${ }^{1} \cdot$ Yoko Okitsu $^{1} \cdot$ Yasushi Onishi $^{1} \cdot$ Ryo Ichinohasama $^{2}$. \\ Hideo Harigae ${ }^{1}$
}

Received: 15 July 2019 / Accepted: 15 October 2019 / Published online: 9 November 2019

(C) Springer-Verlag GmbH Germany, part of Springer Nature 2019

\begin{abstract}
A 62-year-old woman was diagnosed with autoimmune hemolytic anemia (AIHA), with cytology- and culture-negative pleural effusion. AIHA did not improve with steroid therapy. Computerized tomography (CT) detected a left ovarian tumor, which was resected surgically, and AIHA improved temporarily. Pathological examination yielded a diagnosis of diffuse large B-cell lymphoma (DLBCL). Positron emission tomography with CT suggested systemic infiltration of lymphoma. Rituximabcontaining chemotherapy (R-CHOP regimen) yielded rapid decreases in lymphoma lesions and pleural effusion with improvement of AIHA. After six courses of R-CHOP, complete response (CR) was confirmed and AIHA remitted completely. Two months after confirmation of CR, however, lymphoma and AIHA relapsed simultaneously. To our knowledge, this is the first case report describing ovarian DLBCL preceded by AIHA.
\end{abstract}

Keywords Autoimmune hemolytic anemia $\cdot$ Diffuse large B-cell lymphoma $\cdot$ Primary ovarian lymphoma

\section{Introduction}

Autoimmune hemolytic anemia (AIHA) is a disorder caused by destruction of red blood cells by autoantibodies and may be either a primary or a secondary disease. Non-Hodgkin's lymphomas (NHL), such as chronic lymphocytic leukemia (CLL) or angioimmunoblastic T-cell lymphoma (AITL), are wellknown causes of secondary AIHA; diffuse large B-cell lymphoma (DLBCL) is a rare histopathological subtype associated with AIHA $[1,2]$. Case series describing the clinicopathological features of NHL with AIHA have been reported [3, 4]. The diagnosis of AIHA was shown to often precede diagnosis of NHL, at diagnosis the disease is often at an advanced stage, and DLBCL associated with AIHA is refractory to conventional chemotherapy and has poor prognosis.

Primary ovarian lymphoma is a rare disease, although secondary ovarian invasion by lymphoma is not uncommon [5].

Satoshi Ichikawa

satoshi.ichikawa.b4@tohoku.ac.jp

1 Department of Hematology and Rheumatology, Tohoku University Hospital, 1-1 Seiryo-cho Sendai 980-8574 Japan

2 Department of Hematopathology, Tohoku University Hospital, Sendai Japan
According to a report aggregating data regarding primary ovarian lymphoma, DLBCL is the most common histological subtype [6]. In addition, it has been suggested that primary ovarian DLBCL could be an aggressive disease with poor prognosis $[7,8]$.

Here, we report a case of AIHA preceding a diagnosis of primary ovarian DLBCL. We reached a diagnosis of DLBCL through searching for the cause of treatment-resistant AIHA. The importance of investigation of underlying diseases should be emphasized in such cases of refractory AIHA. To our knowledge, this is the first case report describing ovarian DLBCL which was preceded by AIHA.

\section{Case report}

A 62-year-old woman suffering from shortness of breath and easy fatigability for 1 month was referred to our department in January 2017. Chest X-ray examination revealed pleural effusion on the right side, which was both cytology- and culturenegative. Complete blood tests showed macrocytic anemia (hemoglobin $8.8 \mathrm{~g} / \mathrm{dL}$ and mean corpuscular volume 108.6 fL) with increased reticulocytes (11.2\%) and normal white blood cell and platelet counts. Spherocytes are frequently seen on blood smear. Serum biochemical analysis revealed 
elevation of lactic dehydrogenase (395 IU/L) and indirect bilirubin $(0.9 \mathrm{mg} / \mathrm{dL})$. Serum haptoglobin could not be measured, and direct Coombs test was positive. A diagnosis of AIHA was established based on the above findings. Initially, spontaneous improvement of anemia was observed, but anemia later progressed. She was treated with prednisolone (PSL, $0.5-1 \mathrm{mg} / \mathrm{kg} /$ day) beginning in early April; however, the anemia did not improve and the symptoms worsened. For further examination and treatment, she was admitted to hospital in mid-May 2017.

Physical examination revealed anemia and mild jaundice. Hemogram confirmed exacerbation of anemia ( $\mathrm{Hb} 7.2 \mathrm{~g} / \mathrm{dL}$ ) with increased reticulocytes $(18.4 \%)$. Further elevation of serum indirect bilirubin $(1.9 \mathrm{mg} / \mathrm{dL})$ and lactate dehydrogenase (582 IU/L) was also observed. There were no abnormalities in hepatic or renal function. Right pleural effusion was unchanged on chest X-rays (Fig. 1a). Computerized tomography (CT) revealed a large left ovarian mass and a small amount of right pleural effusion (Fig. 1b, c). There was no hepatosplenomegaly, lymphadenopathy, or the other tumor lesions. Magnetic resonance imaging (MRI) also revealed a multinodular and tubular tumor in the left side of the uterus (Fig. 1d, e). It was considered that the ovarian tumor could be associated with AIHA and pleural effusion, and resection of the ovary was performed at our gynecology department. The postoperative course was uneventful, and AIHA improved even with tapering of PSL. She was discharged 16 days after surgery. The clinical course is shown in Fig. 2.

Pathological analysis of the ovarian tumor revealed diffuse infiltration by atypical lymphoid cells with frequent mitosis and apoptosis (Fig. 3a). Necrotic figures were also observed frequently. The atypical cells were immunohistochemically $\mathrm{CD}^{+} 0^{+}$(weak), $\mathrm{CD}^{2}{ }^{+}, \mathrm{CD} 79 \mathrm{a}^{+}, \mathrm{BCL}^{+}, \mathrm{BCL}^{+}$, and $\mathrm{MUM1}^{+}$(Fig. 3b-f). MYC was mostly negative or marginally positive (Fig. 3g). These findings could not confirm the subclassification of germinal center Bcell-like (GCB) or non-GCB immunophenotype definitely. Epstein-Barr virus-encoded small RNA was shown to be negative on in situ hybridization (Fig. 3h). Ki-67 labeling index was as high as $90 \%$ (Fig. 3i). The diagnosis of DLBCL was established based on the above findings. Cytopathological examination of the pleural effusion was performed again 1 month after surgery, and apparent infiltration of abnormal lymphoid cells was observed, which was considered to represent infiltration of DLBCL. Positron emission tomography combined with CT (PET/CT), which was taken 1 month after surgery, revealed multiple extranodal lesions in the right atrium, proximal jejunum, right adrenal gland, and around the left obturator lymph node (Fig. 4). They had not been detected on previous CT taken before surgery. Bone marrow biopsy revealed no apparent infiltration of lymphoma. She did not suffer from B symptoms, and clinical stage was judged as IV-A. The primary lesion site was considered to be the ovary, because the definite tumor lesion was initially detected only on the ovary as shown in Fig. 1.

The patient was readmitted in late June, and chemotherapy with the CHOP regimen (cyclophosphamide $750 \mathrm{mg} / \mathrm{m}^{2}$ on day 1 , doxorubicin $50 \mathrm{mg} / \mathrm{m}^{2}$ on day 1 , vincristine $1.4 \mathrm{mg} / \mathrm{m}^{2}$ on day 1 , and prednisolone $100 \mathrm{mg} /$ body on days $1-5$ in a 21 day cycle) was promptly started. On the day after initiation of CHOP, she experienced hypoxemia and moving difficulties due to bradycardia and hypotension, which were considered due to sinus node dysfunction. It was assumed that her sinus function would be temporarily irritated by the collapse of the lymphoma lesion on the right atrium and hypercytokinemia. We added steroid pulse therapy (methylprednisolone $500 \mathrm{mg}$ for 3 days) with administration of dopamine and red blood cell transfusion. Her circulatory state recovered within 1 week,
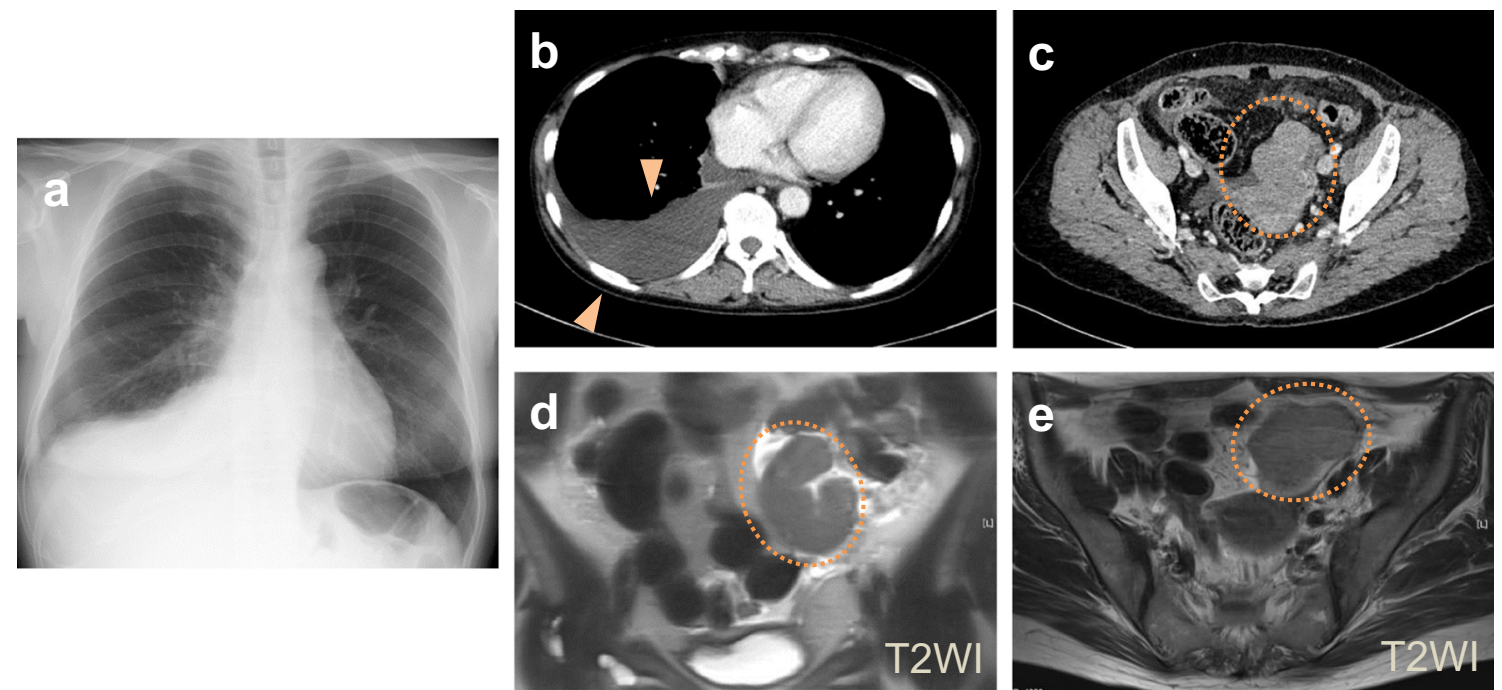

Fig. 1 Radiological findings at detection of the ovarian tumor. Chest Xray examination revealed pleural effusion on the right side (a). CT revealed a large left ovarian mass (b, arrowhead), and a small amount of right pleural effusion (c, circle). MRI (T2-weighted image) revealed a multinodular and tubular tumor in the left side of the uterine body (circle) in coronal (d) and horizontal (e) sections 


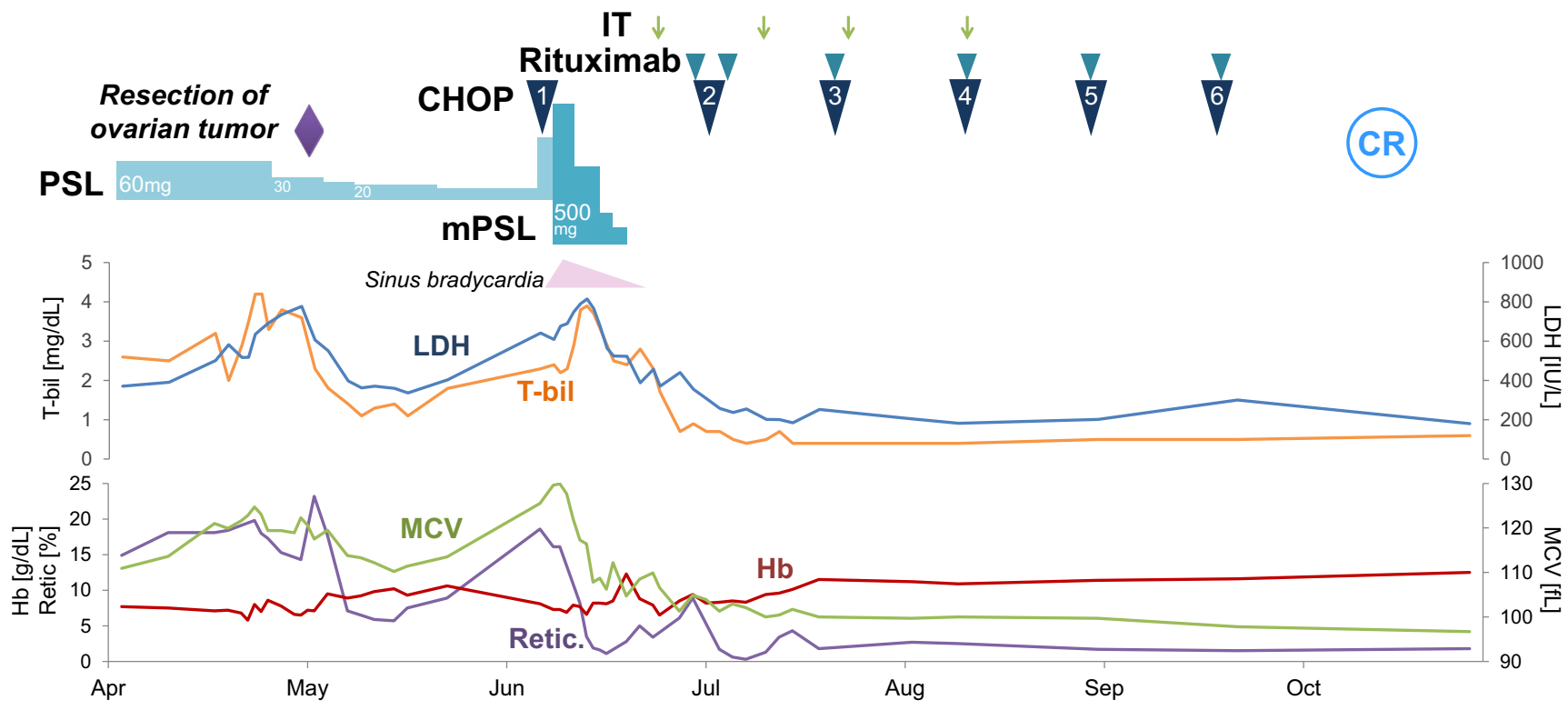

Fig. 2 Clinical course. CHOP cyclophosphamide, doxorubicin, vincristine, prednisolone; $C R$ complete remission; $I T$ intrathecal chemotherapy; $m P S L$ methylprednisolone

and rituximab $\left(375 \mathrm{mg} / \mathrm{m}^{2}\right)$ was also administered (i.e., RCHOP regimen). We also performed intrathecal administration of methotrexate $(15 \mathrm{mg})$ and dexamethasone $(3.3 \mathrm{mg})$ for prophylaxis of central nervous system (CNS) infiltration.
After the first course of R-CHOP, the lymphoma lesions were markedly decreased, and AIHA and the pleural effusion also improved. Thereafter, she received a total of six courses of R$\mathrm{CHOP}$ and four courses of intrathecal chemotherapy on an

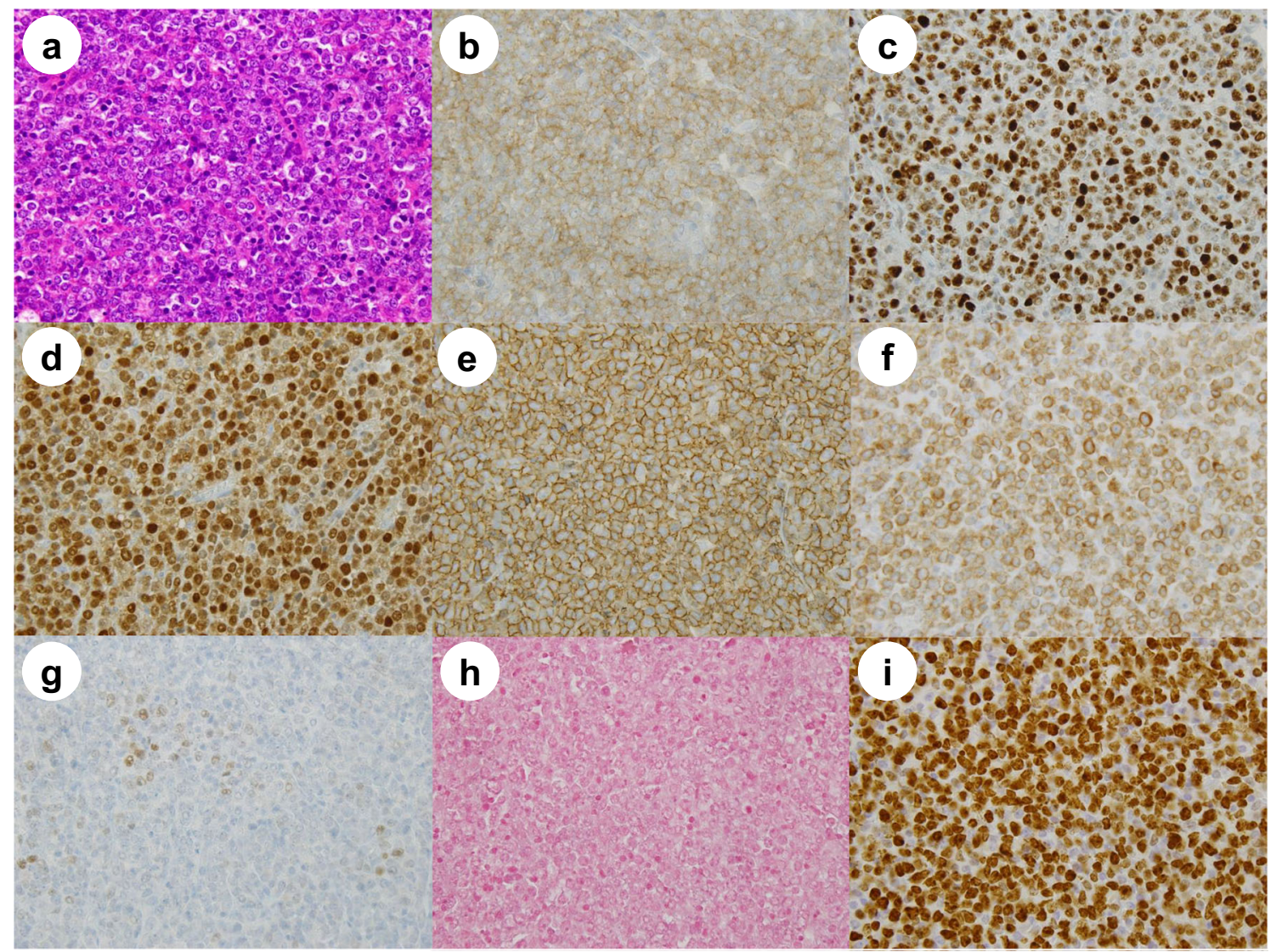

Fig. 3 Histopathological findings of the ovarian tumor. a Hematoxylin-eosin staining. b-i Immunohistochemical findings of CD10 (b), BCL6 (c), MUM1 (d), CD20 (e), BCL2 (f), MYC (g), Epstein-Barr virus-encoded small RNA (in situ hybridization) (h), and Ki-67 (i) 
Fig. 4 PET-CT findings before treatment
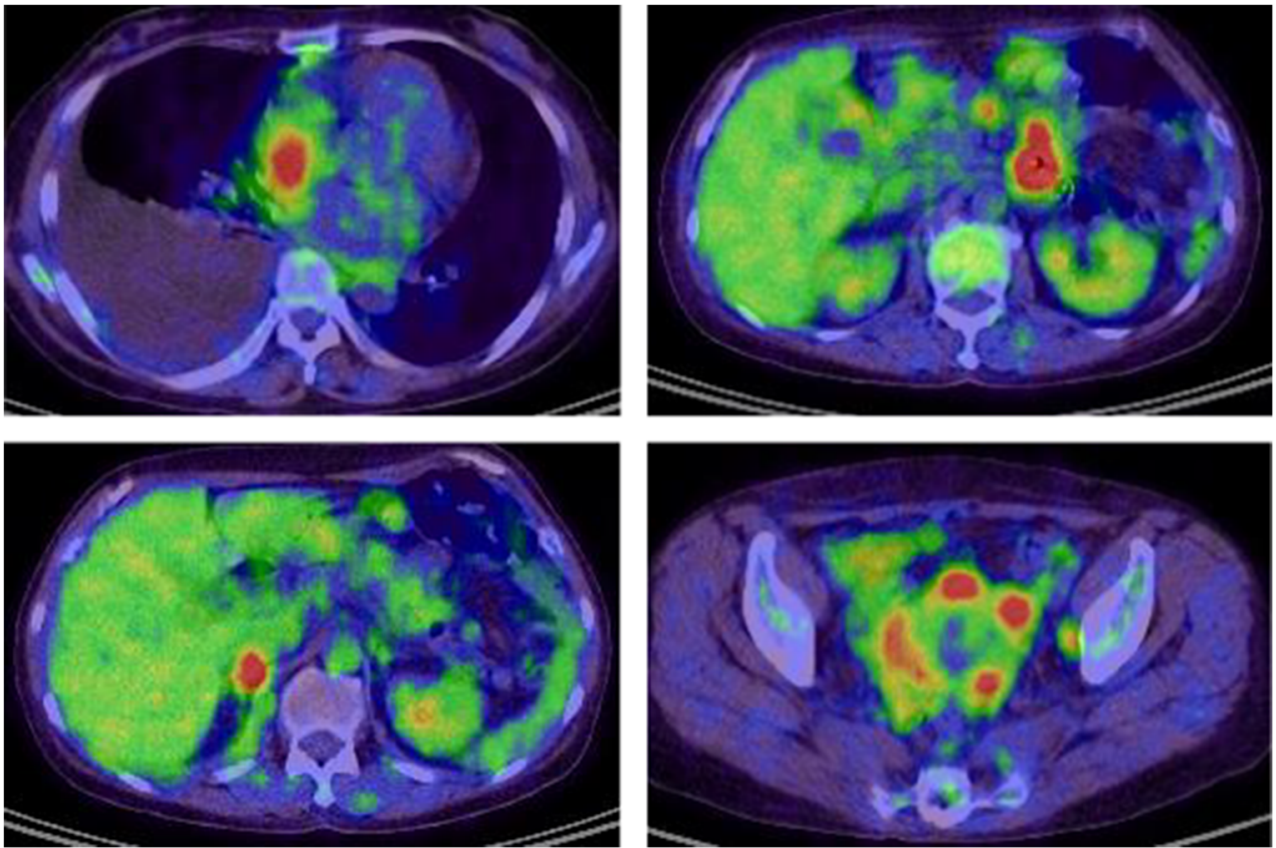

outpatient basis without any cardiovascular problems or exacerbation of anemia. After completion of the above treatment, PET/ CT confirmed complete response (CR). AIHA was also considered to have remitted completely, as serum haptoglobin level was normalized and Coombs test became negative. However, 2 months after confirmation of $\mathrm{CR}$, she experienced simultaneous relapse of DLBCL and AIHA. Several regimens of salvage chemotherapy were performed. However, they did not result in sufficient disease control, and she was transferred to another hospital for palliative care.

\section{Discussion}

NHL, such as CLL and AITL, accounts for about half of all underlying diseases of secondary AIHA, and the association of DLBCL with AIHA is relatively rare $[1,2]$. The pathogenesis of AIHA in NHL has not been elucidated, but it is possible that lymphoma cells could be strongly related to the production of clonal antibodies involved in AIHA. Hauswirth et al. reported four reasons to support this suggestion as follows [4]: First, AIHA shows a good response to effective treatment against lymphoma. Second, there are monoclonal gammopathies in many cases. It can be assumed that these patients had a monoclonal gammopathy with biologically active gamma globulins, and their producing cells could evolve to overt lymphoma. In other cases, AIHA may be associated with autoimmune diseases, which often precede NHL. Third, there is laboratory evidence of the production of antibodies by lymphoma cells [9]. Finally, immunoglobulin VH4-34 gene usage is common in NHL, especially DLBCL, and VH4 family members frequently encode immunoglobulin with autoantibody activity, particularly against red cells. In the present case, the temporary improvement of AIHA after tumor resection may represent direct evidence that DLBCL provoked AIHA. In addition, the synchronous correlation of the disease status of DLBCL and AIHA also supported the above assumption.

The occurrence of AIHA in DLBCL is considered to be a rare phenomenon, and the clinicopathological features remain to be fully elucidated. Sallah et al. [3] described the characteristics of cases of NHL other than CLL and AITL complicated with AIHA in a retrospective cohort study. In their report, 16 cases among the total of 517 cases of NHL were complicated with AIHA, and only 2 cases were diagnosed as DLBCL. Hauswirth et al. [4] described the clinical features of 25 cases of aggressive B-cell lymphoma (22 cases of DLBCL) complicated with AIHA as follows: First, the majority of patients had advanced disease. Second, the timing of diagnosis was variable; AIHA preceded the diagnosis of lymphoma in 9 cases, and the diagnosis of AIHA and lymphoma was simultaneous in 11 cases. Third, chemotherapy was performed in 16 cases and resulted in good response in 13 cases; however, 8 patients died within a short time, and 3 had a recurrence of AIHA. In the present case, DLBCL showed systemic infiltration at the initiation of treatment, and disease relapse was experienced soon after achievement of CR. The above findings suggest that DLBCL complicated with AIHA may be a clinically challenging disease entity.

On the other hand, primary ovarian DLBCL is an extremely rare disease entity, and it could have an aggressive clinical course with poor prognosis. Xiao-Ying et al. described the clinical features and treatment outcomes of 14 cases of primary ovarian NHL [7]. The median age was 45 years, and 13 patients were diagnosed with DLBCL. Seven patients were at limited clinical stage, and seven had advanced disease. All 
patients underwent resection due to pelvic mass at presentation and were treated with chemotherapy after surgery (CHOP in nine cases and R-CHOP in four cases). The median overall survival of the 14 patients was 23.0 months (range 11.5-71.2 months), and all of the patients died. Cao et al. reported the high risk of CNS relapse in patients with primary DLBCL of the female genital tract [8]. Among 323 cases of DLBCL in a single center, there were only 7 cases of primary DLBCL of the female genital tract. Six patients with complete medical data were included in the analysis (two cases with ovarian involvement, two cases with uterine cervical involvement, and two cases with vaginal involvement). Treatment included chemotherapy in five patients and combined chemotherapy and localized radiation in one patient. After a median follow-up of 58 months, four patients showed relapse in the CNS and two died from progressive disease. In recent years, however, it has been reported that rituximab-containing chemotherapy may be effective [10-12]. In the present case, rituximab-containing chemotherapy was highly effective for disease control at least in the short term; however, early relapse occurred. A novel therapeutic strategy is needed to cure such aggressive lymphomas.

In summary, we reported a case of primary ovarian DLBCL diagnosed through investigation to determine the cause of treatment-resistant AIHA. In secondary AIHA based on NHL, the diagnosis of AIHA may precede that of NHL, and in such cases, AIHA is refractory to steroid treatment. It is important to explore the underlying disease in cases of treatment-resistant AIHA. DLBCL complicated with AIHA is rare but could be a clinically formidable condition. Primary ovarian DLBCL is also a rare and challenging disease entity. To our knowledge, this is the first report of primary ovarian DLBCL complicated with AIHA. Further accumulation of clinical experience is necessary to gain a greater understanding of these disease entities.

\section{Compliance with ethical standards}

Conflict of interest The authors declare that they have no competing interests.

\section{References}

1. Packman CH (2015) The clinical pictures of autoimmune hemolytic anemia. Transfus Med Hemother 42:317-324

2. Valent $P$, Lechner $K$ (2008) Diagnosis and treatment of autoimmune haemolytic anaemias in adults: a clinical review. Wien Klin Wochenschr 120:136-151

3. Sallah S, Sigounas G, Vos P, Wan JY, Nguyen NP (2000) Autoimmune hemolytic anemia in patients with non-Hodgkin's lymphoma: characteristics and significance. Ann Oncol 11:15711577

4. Hauswirth AW, Skrabs C, Schutzinger C et al (2007) Autoimmune hemolytic anemias, Evans' syndromes, and pure red cell aplasia in non-Hodgkin lymphomas. Leuk Lymphoma 48:1139-1149

5. Nasioudis D, Kampaktsis PN, Frey M, Witkin SS, Holcomb K (2017) Primary lymphoma of the female genital tract: an analysis of 697 cases. Gynecol Oncol 145:305-309

6. Vang R, Medeiros LJ, Fuller GN, Sarris AH, Deavers M (2001) Non-Hodgkin's lymphoma involving the gynecologic tract: a review of 88 cases. Adv Anat Pathol 8:200-217

7. Zhao XY, Hong XN, Cao JN, Leaw SJ, Guo Y, Li ZT, Chang JH (2011) Clinical features and treatment outcomes of 14 cases of primary ovarian non-Hodgkin's lymphoma: a single-center experience. Med Oncol 28:1559-1564

8. Cao XX, Li J, Zhang W, Duan MH, Shen T, Zhou DB (2014) Patients with primary diffuse large B-cell lymphoma of female genital tract have high risk of central nervous system relapse. Ann Hematol 93:1001-1005

9. Nobuoka A, Sakamaki S, Kogawa K, Fujikawa K, Takahashi M, Hirayama Y, Takayanagi N, Ikeda H, Sekiguchi S, Niitsu Y (1999) A case of malignant lymphoma producing autoantibody against platelet glycoprotein Ib. Int J Hematol 70:200-206

10. Bhartiya R, Kumari N, Mallik M, Singh RV (2016) Primary nonHodgkin's lymphoma of the ovary - a case report. J Clin Diagn Res 10:ED10-ED11

11. Islimye Taskin M, Gokgozoglu L, Kandemir B (2013) Primary ovarian large B-cell lymphoma. Case Rep Obstet Gynecol 2013: 493836

12. Takei T, Uchiyama M (2013) Primary ovarian non-Hodgkin's lymphoma. BMJ Case Rep 2013

Publisher's note Springer Nature remains neutral with regard to jurisdictional claims in published maps and institutional affiliations. 